\title{
Acute Generalized Exanthematous Pustulosis Associated with Tigecycline
}

\author{
Savas Ozturk, Cemal Ustun ${ }^{1}$, Sultan Pehlivan², Haydar Ucak ${ }^{3}$ \\ ${ }^{1}$ Department of Dermatology, Balikesir University Medical Faculty, Balikesir, \\ Departments of ${ }^{2}$ Infectious Diseases and Clinical Microbiology, ${ }^{3}$ Pathology, Ministry of Health Elazig Education and \\ Research Hospital, Elazig, ${ }^{4}$ Department of Dermatology, Dicle University Medical Faculty, Diyarbakir, Turkey
}

Acute generalized exanthematous pustulosis (AGEP) is a severe and rare eruption that develops mostly from factors related to drugs. It is characterized by a fever and a pustular eruption on the erythematous skin with an acute onset and without follicular localization. Etiopathogenesis has not yet been fully explained. Although it is similar to pustular psoriasis, its clinical, historical and histopathological characteristics are different. In this article, we present a case of AGEP associated with tigecycline that developed in a patient followed up in the intensive care unit for three months with an intra-abdominal injury after a trauma and Acinetobacter baumannii infection. (Ann Dermatol 26(2) $246 \sim 249,2014$ )

\section{-Keywords-}

Acute generalized exanthematous pustulosis, Tigecycline

\section{INTRODUCTION}

Acute generalized exanthematous pustulosis (AGEP) is a rare and severe cutaneous reaction with a sudden onset on the erythematous skin characterized by small and sterile pustules, fever and leukocytosis, and a lack of follicular localization ${ }^{1}$. AGEP was first described by Beylot and colleagues ${ }^{2}$ in France as a generalized pustular eruption with

Received May 13, 2011, Revised November 16, 2011, Accepted for publication November 18, 2011

Corresponding author: Savas Ozturk, Department of Dermatology, Balikesir University Medical Faculty, 10100 Balikesir, Turkey. Tel: 90 50565963 33, Fax: 9042423810 00, E-mail: drsozturk@gmail.com

This is an Open Access article distributed under the terms of the Creative Commons Attribution Non-Commercial License (http:// creativecommons.org/licenses/by-nc/3.0) which permits unrestricted non-commercial use, distribution, and reproduction in any medium, provided the original work is properly cited. a sudden onset in the patients without a history of psoriasis. Although the etiopathogenesis has not yet been explained fully, drugs are held responsible in $90 \%$ of the cases. Of these drugs, aminopenicillins and macrolides are accused most frequently.

Apart from the adverse reaction to drugs, the condition has been seen after viral infections, allergic reactions to foods, mercury hypersensitivity, an exposure to radiation, and spider bits ${ }^{2,3}$. The different diagnosis of AGEP should be made with generalized pustular psoriasis, subcorneal pustular dermatosis, Immunoglobulin A dermatosis, Drug Reaction with Eosinophilia and Systemic Symptoms syndrome and toxic epidermal necrolysis ${ }^{4}$.

The disease is generally self-limited when the causative factor is eliminated; and cure can be ensured with a shortterm systemic steroids and a symptomatic treatment ${ }^{5}$.

Tigecycline is a novel, broad-spectrum antimicrobial in the glycycline group particularly effective against the nosocomial multi-drug-resistant strains, including Acinetobacter baumannii infections. The most adverse effects of tigecycline are nausea, vomiting and diarrhea ${ }^{6-8}$.

AGEP associated with tigecycline has not been reported in literature; but it has been reported that this drug can cause various cutaneous reactions ${ }^{1}$.

This article presents a case of AGEP due to tigecycline.

\section{CASE REPORT}

The eruption being evaluated appeared in a 39-year-old male being treated in the intensive care unit for a ventilatorassociated pneumonia caused by $A$. baumannii. The patient had been under a follow-up for the past 45 days with confusion and intra-abdominal trauma after being involved in a traffic accident as a passenger. For the last 4 days, he had progressively started pustular eruptions on the erythe- 


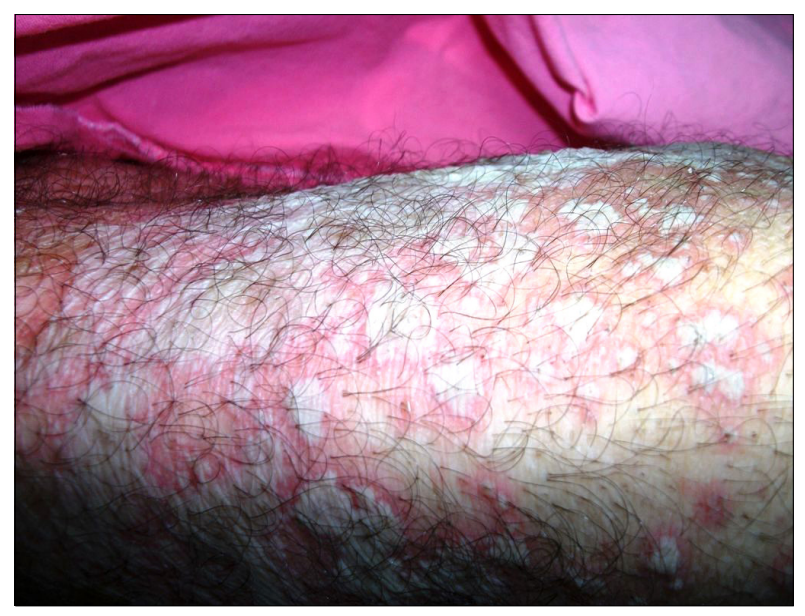

Fig. 1. Revealed numerous pustules on erythematous areas that tended to unite and did not display a follicular localization on the legs.

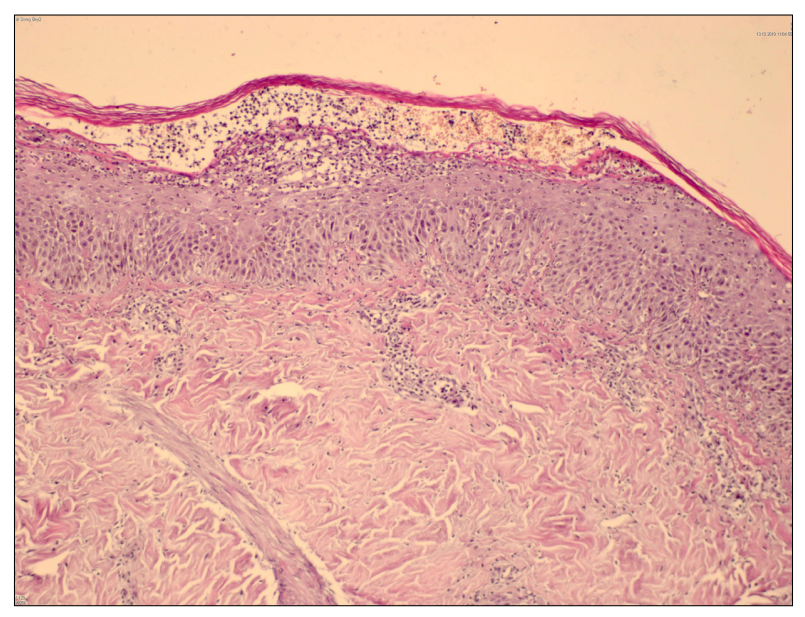

Fig. 2. Subcorneal and intraepidermal pustules, widespread spongiosis, neutrophil and histiocyte infiltration around vessels in the papillary dermis and exocytosis of eosinophils $(\mathrm{H} \& \mathrm{E}, \times 200)$.

matous areas, which started on the facial and the neck region, and then, rapidly spread to the upper and the lower extremity. The patient's history recorded that he had registered a fever of $39.5^{\circ} \mathrm{C} /$ axillary for the last 1 week. Upon finding an $A$. baumannii growth in the patient's tracheal aspirate culture, tigecycline $50 \mathrm{mg}$ intravenous, twice daily, was added to his treatment for 5 days for the ventilator-associated nosocomial pneumonia. The patient's history showed that no other new drugs had been started during the past 15 days. In the clinical management, the empirical tigecycline treatment initially was started due to sepsis. After two days of this treatment, mild erupted lesions occurred on the skin of the patient, which were considered to result from tigecycline and the treatment was stopped. After 15 days of stopped tigecycline, A.

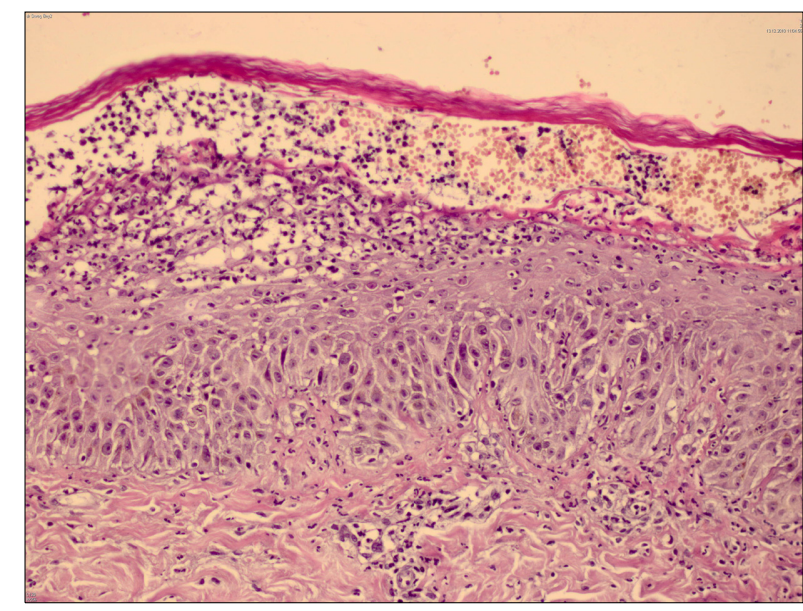

Fig. 3. Subcorneal and intraepidermal pustules, widespread spongiosis, neutrophil and histiocyte infiltration around vessels in the papillary dermis and exocytosis of eosinophils $(H \& E, \times 400)$.

baumanii was recovered from two tracheal aspirate cultures. The tigecycline treatment was started again by another clinician. Generalized erupted lesions occurred on the skin of the patient after 4 days of the tigecycline treatment. Thus, this condition was considered a therapeutic provocation due to the tigecycline.

Erythema appeared on the patient's face and the neck about 12 hours after the start of the tigecycline, and the pustules appeared later. The dermatologic examination revealed numerous pustules on the face, the neck and the legs, as well as on the erythematous areas that tended to unite and did not display a follicular localization (Fig. 1).

The oral examination found no pathologies. Psoriasis was negative in the patient's personal history and in the history taken from his family. Furthermore, the family history showed no known allergies.

The patient had fever and leukocytosis. A culture taken from the pustules was negative.

Based on the pre-diagnoses of AGEP and pustular psoriasis, a 4-mm punch biopsy was taken from the patient. The histopathologic examination found sub-corneal and intraepidermal pustules, widespread spongiosis, neutrophil and histiocyte infiltration around the vessels in the papillary dermis and exocytosis of eosinophils (Fig. 2, 3).

The patient was diagnosed with AGEP according to the existing clinical and histopathologic findings. The tigecycline treatment was stopped and an intravenous methylprednisolon $60 \mathrm{mg} / \mathrm{d}$ was administered for three days, together with local moisturizers and topical steroids.

The appearance of new pustules stopped, and the healing continued with an exfoliation. No dermatologic pathologies remained on the patient for 15 days, except on the facial area. The patient was followed in the intensive care unit 
for about 3 months, until he died of sepsis and a multiorgan failure unrelated to the earlier AGEP.

\section{DISCUSSION}

AGEP is an entity without a very long past history. In the literature, it was defined in 1968 in five patients who developed a drug-related pustular eruption without a history of psoriasis. At first, it was considered a subtype of pustular psoriasis.

In 1980, AGEP was evaluated for the first time as a separate entity $^{2}$. It can be differentiated from pustular psoriasis by its histopathologic characteristics, its short course, the lack of psoriasis in the anamnesis and the involvement mostly of flexor surfaces ${ }^{5}$.

Drugs (particularly antibiotics) are held to be mainly responsible for this etiopathogenesis. The literature reports that drug-related AGEP develops in less than 24 hours ${ }^{1,5}$. There are also reports in the literature of cases developing after acute viral infections and spider bites.

Sixty-three separate drugs have been reported as the cause of $\mathrm{AGEP}^{9}$. In a retrospective series of 36 patients, aminopenicillins, like amoxicillin, were held to be most responsible, with macrolides, meropenem, clopidogrel, herbal medicines, aspirin, chemotherapeutical agents and radiocontrast materials following.

In the same study, only 3 patients who developed AGEP had any history of allergic reactions to drugs ${ }^{2}$. Our patient also had no known past drug allergy.

Since tigecycline is similar to minocycline, which is a member of the tetracycline family of drugs, potential adverse effects may include e-hepatitis, pancreatitis, vertigo, hearing loss, lupus erythematosus-like syndrome, and hyperpigmentation ${ }^{6}$. Various cutaneous reactions have been defined with tigecycline; but no AGEP cases have been reported ${ }^{1}$.

We consider that the development of AGEP is associated with the use of tigecycline, since the typical clinical findings appeared 12 hours after the administration of tigecycline. The morphology of the lesions, fever, leukocytosis and the rapid healing after stopping the drug makes the clinical diagnosis of AGEP relatively easy ${ }^{2}$. The eruption typically starts on the erythematous skin of the face and the intertriginous areas. The sterile pustular eruption spreads rapidly and tends to unite.

Purpura, vesicle formation, bullae, and erythema multiforme-like lesions have been described in some AGEP cases $^{9,10}$. Fever, leukocytosis and mild eosinophilia accompanies most of the cases, and there is a mucosal involvement in about $20 \%$ of the cases. The literature reports the Involvement of the internal organs and the mortality rate at about $5 \%{ }^{2,11}$.
In our case, the onset of the eruption was compatible with the literature. There was no mucosal involvement and no lesions were seen other than the sterile pustules on the erythematous skin.

Histopathologically, dermal edema and spongiosis appear as the background in AGEP, along with perivascular eosinophils and subcorneal or intra-epidermal pustules. The lack of hyperplasia and achantosis are important when differentiating AGEP from pustular psoriasis ${ }^{2,4,5}$. The presence of histopathological findings typical for AGEP is helpful for making different diagnosis.

It is not always easy to determine the causative factor in the AGEP cases; the factors could not be found in 13 cases out of a retrospective series of 36 cases $^{2}$.

The patch test is the method most frequently-used to determine the causative factor in cutaneous drug reactions. In AGEP cases, the ratio of sensitivity to a patch test is about $50 \%$. The literature recommends using $10 \%$ to $30 \%$ concentration of the suspected drugs in the test patch ${ }^{12,13}$. Since our patient was in the intensive care unit and his general medical condition was poor, we did not perform a patch test with tigecycline.

In the end, we determined that tigecycline may cause AGEP. Since AGEP is a self-limiting condition, eliminating the causative factor and administering topical steroids and short-duration systemic steroids as the treatment may be sufficient.

In our case, the reaction limited itself after we stopped administering tigecycline. After the use of a systemic steroid for three days, a full recovery took about 14 days, which was consistent with the literature.

\section{REFERENCES}

1. Litt JZ. Drug eruptions \& reactions manual. 16th ed. New York: Informa Healthcare, 2010:572.

2. Beylot C, Bioulac P, Doutre MS. Acute generalized exanthematic pustuloses (four cases). Ann Dermatol Venerol 1980; 107:37-48.

3. Sidoroff A, Dunant A, Viboud C, Halevy S, Bavinck JN, Naldi $L$, et al. Risk factors for acute generalized exanthematous pustulosis (AGEP)-results of a multinational casecontrol study (EuroSCAR). Br J Dermatol 2007;157:989-996.

4. Sidoroff A, Halevy S, Bavinck JN, Vaillant L, Roujeau JC. Acute generalized exanthematous pustulosis (AGEP)--a clinical reaction pattern. J Cutan Pathol 2001;28:113-119.

5. Won JH, Yun SJ, Kim SJ, Lee SC, Won YH, Lee JB. A case of acute generalized exanthematous pustulosis possibly induced by ritodrine. Ann Dermatol 2009;21:323-325.

6. Stein GE, Craig WA. Tigecycline: a critical analysis. Clin Infect Dis 2006;43:518-524.

7. Nursel Ç, Murat A. Tigesiklin. ANKEM Derg 2007;21:2933. 
8. Bassetti M, Nicolini L, Repetto E, Righi E, Del Bono V, Viscoli C. Tigecycline use in serious nosocomial infections: a drug use evaluation. BMC Infect Dis 2010;10:287.

9. Khalel MH, Fattah Saleh SA, F El-Gamal AH, Najem N. Acute generalized exanthematous pustulosis: an unusual side effect of meropenem. Indian J Dermatol 2010;55: 176-177.

10. Makris M, Spanoudaki N, Giannoula F, Chliva C, Antoniadou A, Kalogeromitros D. Acute generalized exanthematous pustulosis (AGEP) triggered by a spider bite. Allergol Int
2009;58:301-303.

11. Yüksek J, Sezer E. Atypical drug eruptions: review. T Clin J Dermatol 2008;18:85-90.

12. Wolkenstein $\mathrm{P}$, Chosidow $\mathrm{O}$, Fléchet $\mathrm{ML}$, Robbiola $\mathrm{O}$, Paul $M$, Dumé $L$, et al. Patch testing in severe cutaneous adverse drug reactions, including Stevens-Johnson syndrome and toxic epidermal necrolysis. Contact Dermat 1996;35:234236.

13. Barbaud A. Drug patch testing in systemic cutaneous drug allergy. Toxicol 2005;209:209-216. 